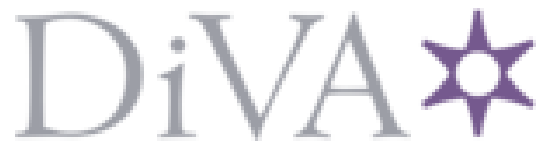

http://www.diva-portal.org

Preprint

This is the submitted version of a paper presented at 2017 IEEE International Symposium on Information Theory, ISIT 2017, Aachen, Germany, 25 June 2017 through 30 June 2017.

Citation for the original published paper:

Vu, M T., Oechtering, T J., Skoglund, M. (2017)

Hierarchical identification with pre-processing

In: 2017 IEEE International Symposium on Information Theory (ISIT), 8007029 (pp.

2746-2750). Institute of Electrical and Electronics Engineers (IEEE)

IEEE International Symposium on Information Theory - Proceedings

https://doi.org/10.1109/ISIT.2017.8007029

N.B. When citing this work, cite the original published paper.

The work was supported by the Swedish Research Council (VR) project under Grant 2016-03853.

Permanent link to this version:

http://urn.kb.se/resolve?urn=urn:nbn:se:kth:diva-223032 


\title{
Hierarchical Identification with Pre-processing
}

\author{
Minh Thanh Vu, Tobias J. Oechtering, Mikael Skoglund \\ Department of Information Science and Engineering \\ KTH-Royal Institute of Technology
}

\begin{abstract}
We study a two-stage identification problem with pre-processing to enable efficient data retrieval and reconstruction. The first stage outputs a list of compatible users to the second stage which uses it to return the exact user identity with a corresponding reconstruction sequence. The ratedistortion region is characterized. A connection to a two observer identification problem is also studied.
\end{abstract}

\section{INTRODUCTION}

The blooming numbers of smart devices lead to an increase in high-dimensional contents such as videos or audios. Nowadays, large parts of these contents are stored in databases of data centers. Hence, it is motivated to study efficient storage schemes that also allow data to be retrieved and reconstructed with low complexity. Additionally, there are many image or video processing applications in eHealth, IoT, etc., where an efficient identification is crucial. However, using highdimensional observations directly puts a heavy toll on the system. This motivates a pre-processing procedure to reduce costs at some steps. The pre-processing can also be done for other purposes such as enhancing user privacy at these steps.

The identification problem was first studied by Willems in [1], where he characterized the identification capacity for biometric systems. The compression and distortion aspects were taken into accounts in [2] and [3], where the trade-offs between compression and identification rates, and compression-distortion-capacity, respectively, were provided. Clustering was considered in [4], [5], and [6] as a method to improve the search speed, where in the enrollment phase each user's data are put into (possibly multiple) clusters.

In this work we study an identification problem in which we assume only one data cluster with two storage nodes in the system. The focus is, hence, to study the capacity-listcompression-distortion trade-off for the given cluster. In our setup each data sequence $x^{n}(i)$, which corresponds to the $i$-th user is compressed and stored in two layers. We note that a user is actually represented by a data entry which does not have to be an actual person. The first layer stores some representative features of the sequence as in [7], which is retrieved frequently by the system or other applications. The second layer contains refinement information. This information layer helps to identify the user exactly and reconstruct the corresponding data sequence. However, querying information in the second layer directly is costly and therefore the system needs to limit the number of queries. An observation $y^{n}$ is provided to the processing unit which needs to return the correct user's identity and its corresponding reconstruction sequence. To facilitate the processing time and power, the

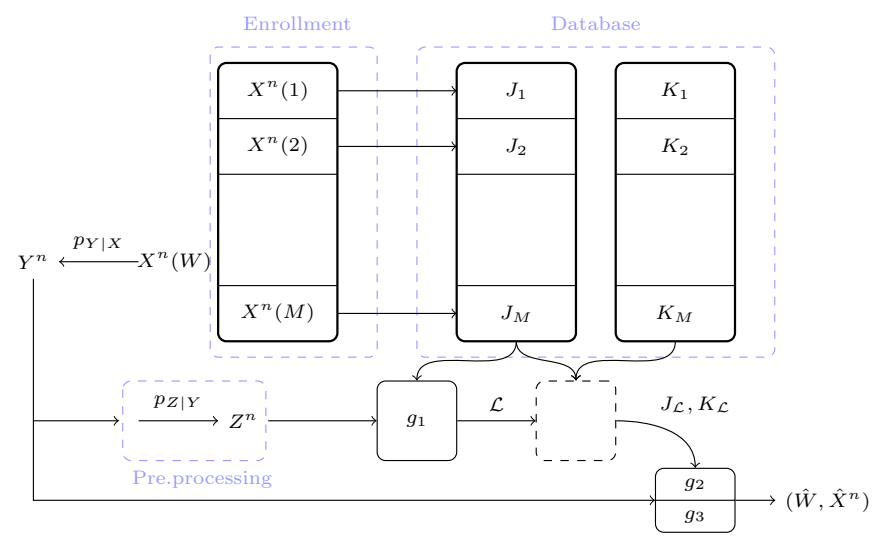

Fig. 1. An overview of the two stage identification system. We assume that there always exists a user $W$ which has been enrolled previously and to which the observation $Y^{n}$ is the output of a memoryless channel $p_{Y \mid X}$ with the input $X^{n}(W)$. Furthermore, $W$ is uniformly distributed over $[1: M]$ and independent of users' data. The first and second layer information are represented by the collections $\left\{J_{i}\right\}_{i=1}^{M}$ and $\left\{K_{i}\right\}_{i=1}^{M}$, respectively.

observation is first passed through a fixed channel, which can be thought of as a feature extraction operation. The processed observation is then compared with the information in the first layer, which results in a list of compatible users, $\mathcal{L}$. Then, the processing unit retrieves the information contained in both layers for all users in the list, $\left(j_{\mathcal{L}}, k_{\mathcal{L}}\right)$. The retrieved information is then combined with observation $y^{n}$ to idenfity the correct user. Finally, the system outputs the corresponding reconstruction sequence of the identified user.

\section{Problem Formulation}

The letter-wise distortion measure is a bounded mapping of the form $d: \mathcal{X} \times \hat{\mathcal{X}} \rightarrow\left[0, d_{\max }\right]$. With abuse of notation, the sequence distortion measure is defined as

$$
d\left(x^{n}, \hat{x}^{n}\right)=\frac{1}{n} \sum_{i=1}^{n} d\left(x_{i}, \hat{x}_{i}\right)
$$

The strongly typical set is denoted by $\mathcal{T}_{\epsilon}^{n}$. For a set $\mathcal{A},|\mathcal{A}|$ denotes its cardinality.

The (big) data $x^{n}(i)$ generated from the memoryless source with distribution $p_{X}$, where $i \in \mathcal{M}$ with $M=|\mathcal{M}|$, is compressed and stored hierarchically in two layers described by messages $\left(\left\{j_{i}\right\}_{i=1}^{M},\left\{k_{i}\right\}_{i=1}^{M}\right)$, where each $j_{i}$ stores the correspondingly frequently accessed information. These can be described by (stochastic) mappings

$$
\phi_{k}: \mathcal{X}^{n} \rightarrow \mathcal{M}_{k}, k=1,2 .
$$


We denote database indices $\phi_{1}\left(x^{n}(i)\right)$ and $\phi_{2}\left(x^{n}(i)\right)$ as $j_{i} \in$ $\mathcal{M}_{1}$ and $k_{i} \in \mathcal{M}_{2}$ for all $i \in \mathcal{M}$.

An observer obtains information $y^{n}$ about an user in the database from the output of the memoryless channel $p_{Y \mid X}$ with input $x^{n}(w)$, where $w$ is an instance of a uniformly distributed random variable $W$ over the set $\mathcal{M}$, which is independent of the users' data. The observer sends $y^{n}$ to a processing center to identify $w$ and obtain a reconstruction of $x^{n}(w)$ within the distortion $D$.

In the processing center, the observation $y^{n}$ is first passed through a fixed channel $p_{Z \mid Y}$ to produce a noisy version $z^{n}$, which can be linked to a quantization or a feature extraction process. Then, based on $z^{n}$ and the first layer database $\left\{j_{i}\right\}_{i=1}^{M}$, a list $\mathcal{L}$ of possible matching indices of a given size, is produced. This action can be described by a function

$$
\begin{aligned}
g_{1}: \mathcal{Z}^{n} \times \mathcal{M}_{1}^{M} & \rightarrow 2^{\mathcal{M}}, \\
\mathcal{L} & =g_{1}\left(z^{n},\left\{j_{i}\right\}_{i=1}^{M}\right),|\mathcal{L}| \leq 2^{n \Delta} .
\end{aligned}
$$

In the second stage the processing unit returns an estimate of the index $\hat{w}$ and a reconstruction sequence $\hat{x}^{n}$ of $x^{n}(w)$, which are outputs of deterministic functions $g_{2}(\cdot)$ and $g_{3}(\cdot)$, where

$$
\hat{w}=g_{2}\left(y^{n}, j_{\mathcal{L}}, k_{\mathcal{L}}\right),
$$

with $j_{\mathcal{L}}=\left\{j_{i} \mid i \in \mathcal{L}\right\}$, and similarly for $k_{\mathcal{L}}$, and

$$
\begin{aligned}
g_{3}: \mathcal{Y}^{n} \times \mathcal{M}_{1} \times \mathcal{M}_{2} & \rightarrow \hat{\mathcal{X}}_{n} \\
\hat{x}^{n} & =g_{3}\left(y^{n}, j_{\hat{w}}, k_{\hat{w}}\right) .
\end{aligned}
$$

Note that $j_{\mathcal{L}}$ and $k_{\mathcal{L}}$ can be multisets.

Definition 1. A data processing scheme of length $n$ consists of enrollment mappings $\left\{\phi_{k}\right\}_{k=1}^{2}$ and processing mappings $\left\{g_{k}\right\}_{k=1}^{3}$.

Definition 2. A rate-distortion tuple $\left(R, R_{1}, R_{2}, R_{L}, D\right)$ is achievable if for every $\epsilon>0$, there exists a data processing scheme of length $n$ such that

$$
\begin{aligned}
\frac{1}{n} \log M & >R-\epsilon, \quad \frac{1}{n} \log \left|\mathcal{M}_{1}\right|<R_{1}+\epsilon \\
\frac{1}{n} \log \left|\mathcal{M}_{2}\right| & <R_{2}+\epsilon, \quad \Delta<R_{L}+\epsilon, \quad \operatorname{Pr}(W \notin \mathcal{L})<\epsilon, \\
\operatorname{Pr}(W \neq \hat{W}) & <\epsilon, \quad \mathbb{E}\left[d\left(X^{n}, \hat{X}^{n}\right)\right]<D+\epsilon,
\end{aligned}
$$

for all sufficiently large $n$. The set of all achievable tuples is denoted by $\mathcal{R}$.

Definition 3. Let $\mathcal{R}^{\star}$ be the collection of tuples $\left(R, R_{1}, R_{2}, R_{L}, D\right)$ such that there exist random variables $U$ and $V$ defined on finite alphabets $\mathcal{U}$ and $\mathcal{V}$ which satisfy

$$
|\mathcal{U}| \leq|\mathcal{X}|+5,|\mathcal{V}| \leq(|\mathcal{X}|+5)(|\mathcal{X}|+2)
$$

and a deterministic reconstruction mapping $f: \mathcal{U} \times \mathcal{V} \times \mathcal{Y} \rightarrow \hat{\mathcal{X}}$ such that the followings expressions are fulfilled:

$$
\begin{array}{r}
U-V-X-Y-Z, \\
R_{1} \geq I(X ; U),
\end{array}
$$

$$
\begin{aligned}
R_{1}+R_{2} & \geq I(X ; U)+I(X ; V \mid U, Y), \\
R_{1}+R_{2}-R & \geq I(X ; U, V \mid Y), \\
R & \leq \min \left\{R_{L}+I(Z ; U), I(Y ; U, V)\right\}, \\
D & \geq \mathbb{E}[d(X, f(U, V, Y))] .
\end{aligned}
$$

The above definitions imply that both $\mathcal{R}$ and $\mathcal{R}^{\star}$ are closed sets.

Theorem 1. For a given pre-processing strategy $p_{Z \mid Y}$, memoryless data source $p_{X}$ and observation model $p_{Y \mid X}$, the ratedistortion region for our setting is given by

$$
\mathcal{R}=\mathcal{R}^{\star} .
$$

\section{PROOF}

\section{A. Achievability}

Assume that the set $\mathcal{R}^{\star}$ is not empty, fix a conditional pmf $p_{U V \mid X}$ where $U-V-X$ and a deterministic reconstruction mapping $f$ such that

$$
\mathbb{E}[d(X, f(U, V, Y))] \leq D,
$$

Additionally, for an $\epsilon>0$ we assume that the number of enrolled users is given by $M=2^{n \hat{R}}$ where $\hat{R}=R-\epsilon / 2$ and the list size is $\hat{\Delta}=R_{L}+\epsilon / 2$. Also let $\hat{R}_{U}=R_{1}+\epsilon / 2$, $\hat{R}_{V}=R_{2}+\epsilon / 2$ and $\hat{R}_{V}^{\prime}=R_{V}^{\prime}-\epsilon / 4$ be the actual code rates. Codebook generation: Generate $2^{n \hat{R}_{U}}$ iid codewords $u^{n}(j)$ according to the marginal distribution $p_{U}$. For each $j$, we draw $2^{n\left(\hat{R}_{V}+\hat{R}_{V}^{\prime}\right)}$ codewords $v^{n}(j, k, l)$ iid via the conditional distribution $p_{V \mid U}$, where $k \in\left[1: 2^{n \hat{R}_{V}}\right]$ and $l \in\left[1: 2^{n \hat{R}_{V}^{\prime}}\right]$. The same codebook is known in the whole system.

Enrollment: For each user index $i \in \mathcal{M}$, a codeword $u^{n}\left(j_{i}\right)$ is looked for such that $\left(x^{n}(i), u^{n}\left(j_{i}\right)\right) \in \mathcal{T}_{\epsilon}^{n}$. The chosen $j_{i}$ is stored in the first layer. Next, a codeword $v^{n}\left(j_{i}, k_{i}, l_{i}\right)$ is searched for such that

$$
\left(x^{n}(i), u^{n}\left(j_{i}\right), v^{n}\left(j_{i}, k_{i}, l_{i}\right)\right) \in \mathcal{T}_{\epsilon}^{n} .
$$

The chosen index $k_{i}$ is stored in the second layer. We note that in both steps if there is more than one suitable index, we select one of them uniformly at random. Otherwise, an index is selected from the corresponding set uniformly at random.

Identification and Reconstruction: The observation $y^{n}$ is first passed through the memoryless channel $p_{Z \mid Y}$ to produce $z^{n}$ which is used in the first stage of our identification and reconstruction process.

First stage: We look for all indices $i \in \mathcal{M}$ such that

$$
\left(z^{n}, u^{n}\left(j_{i}\right)\right) \in \mathcal{T}_{\epsilon}^{n},
$$

and put them into the list $\mathcal{L}$. If there are more than $2^{n \hat{\Delta}}$ suitable indices then we output a list $\mathcal{L}^{\prime}$ that consists of the first $2^{n \hat{\Delta}}$ elements of $\mathcal{L}$. Additionally, the first stage sends an error message to the second stage. In this way, our list always meets the size constraint in (3).

Second stage: A unique pair $\left(\hat{w}, \hat{l}^{\prime}\right)$ where $\hat{w} \in \mathcal{L}$, is searched for such that

$$
\left(y^{n}, u^{n}\left(j_{\hat{w}}\right), v^{n}\left(j_{\hat{w}}, k_{\hat{w}}, \hat{l}^{\prime}\right)\right) \in \mathcal{T}_{\epsilon}^{n} .
$$


The reconstruction sequence is given as $\hat{x}_{\tau}=$ $f\left(u_{\tau}\left(j_{\hat{w}}\right), v_{\tau}\left(j_{\hat{w}}, k_{\hat{w}}, \hat{l}^{\prime}\right), y_{\tau}\right)$ for all $\tau=\left[\begin{array}{lll}1 & :\end{array}\right]$. When the uniqueness or the existence is violated, the system declares an error. If an error is thrown, the system outputs an arbitrary reconstruction sequence.

Analysis: Let $J_{i}$ and $K_{i}, i \in \mathcal{M}$, be the stored indices for the $i$-th user. Furthermore, let $\mathcal{L}$ be the list of indices $i \in \mathcal{M}$ that satisfy (12) in the first stage of the identification process. Consider the following events

$$
\begin{aligned}
& \mathcal{E}_{u}=\left\{\left(X^{n}(W), U^{n}\left(J_{W}\right)\right) \notin \mathcal{T}_{\epsilon}^{n}\right\}, \\
& \mathcal{E}_{v}=\left\{\left(X^{n}(W), U^{n}\left(J_{W}\right), V^{n}\left(J_{W}, K_{W}, L_{W}\right)\right) \notin \mathcal{T}_{\epsilon}^{n}\right\}, \mathcal{E}_{y z}=\left\{\left(Y^{n}, Z^{n}, X^{n}(W), U^{n}\left(J_{W}\right),\right.\right. \\
&\left.\left.V^{n}\left(J_{W}, K_{W}, L_{W}\right)\right) \notin \mathcal{T}_{\epsilon}^{n}\right\}, \\
& \mathcal{E}_{1}=\left\{|\mathcal{L}|>2^{n \hat{\Delta}}\right\}, \\
& \mathcal{E}_{2}=\left\{\exists l \neq L_{W},\left(Y^{n}, U^{n}\left(J_{W}\right), V^{n}\left(J_{W}, K_{W}, l\right)\right) \in \mathcal{T}_{\epsilon}^{n}\right\}, \\
& \mathcal{E}_{3}=\left\{\exists\left(w^{\prime}, l_{w^{\prime}}\right), w^{\prime} \neq W, w^{\prime} \in \mathcal{L},\left(Y^{n}, U^{n}\left(J_{w^{\prime}}\right),\right.\right. \\
&\left.\left.V^{n}\left(J_{w^{\prime}}, K_{w^{\prime}}, l_{w^{\prime}}\right)\right) \in \mathcal{T}_{\epsilon}^{n}\right\} .
\end{aligned}
$$

Define

$$
\mathcal{E}=\mathcal{E}_{u} \cup \mathcal{E}_{v} \cup \mathcal{E}_{y z} \bigcup_{i=1}^{3} \mathcal{E}_{i},
$$

to be the event that summarizes all "errors." By the covering lemma [8, Lemma 3.3] we obtain

$$
\operatorname{Pr}\left(\mathcal{E}_{u}\right)=\frac{1}{M} \sum_{i} \operatorname{Pr}\left(\left(X^{n}(i), U^{n}\left(J_{i}\right)\right) \notin \mathcal{T}_{\epsilon}^{n}\right) \rightarrow 0
$$

if $\hat{R}_{U}>I(X ; U)+\gamma_{n}$, where $\gamma_{n}>0$ and $\gamma_{n} \rightarrow 0$ as $n \rightarrow$ $\infty$. Similarly, we have $\operatorname{Pr}\left(\mathcal{E}_{u}^{c} \cap E_{v}\right) \rightarrow 0$ if $\hat{R}_{V}+\hat{R}_{V}^{\prime}>$ $I(X ; V \mid U)+\gamma_{n}$. Due to the Markov lemma [8, p.27]

$$
\operatorname{Pr}\left(\mathcal{E}_{u}^{c} \cap \mathcal{E}_{v}^{c} \cap \mathcal{E}_{y z}\right) \rightarrow 0 .
$$

This implies that $\operatorname{Pr}\{W \notin \mathcal{L}\} \rightarrow 0$ as $n \rightarrow \infty$.

Due to symmetry it is sufficient to condition on the event $\{W=1\}$. Following the analysis in [8, Section 11.3] we have

$$
\operatorname{Pr}\left(\mathcal{E}_{2} \mid W=1\right) \rightarrow 0
$$

as $n \rightarrow \infty$ if $\hat{R}_{V}^{\prime}<I(Y ; V \mid U)-\gamma_{n}$. We focus on the two remaining events $\mathcal{E}_{1}$ and $\mathcal{E}_{3}$. For each $i \in \mathcal{M}$ define random variable

$$
B_{i}= \begin{cases}1, & \text { if }\left(Z^{n}, U^{n}\left(J_{i}\right)\right) \in \mathcal{T}_{\epsilon}^{n} \\ 0, & \text { otherwise }\end{cases}
$$

Note that $\operatorname{Pr}\left(B_{1}=1 \mid W=1\right) \rightarrow 1$ as $n \rightarrow \infty$. Hence, it is sufficient to consider the following probability

$$
\begin{aligned}
& \operatorname{Pr}\left(B_{1}=1,|\mathcal{L}|>2^{n \hat{\Delta}} \mid W=1\right) \\
& =\operatorname{Pr}\left\{B_{1}=1, \sum_{i=1}^{2^{n \hat{R}}} B_{i}>2^{n \hat{\Delta}} \mid W=1\right\}
\end{aligned}
$$

$$
\begin{aligned}
& \leq \operatorname{Pr}\left\{\sum_{i=2}^{2^{n \hat{R}}} B_{i}>2^{n \hat{\Delta}}-1 \mid W=1\right\} \\
& \leq \frac{\sum_{i=2}^{2^{n \hat{R}}} \mathbb{E}\left[B_{i} \mid W=1\right]}{2^{n \hat{\Delta}}-1} \leq 2^{n(\hat{R}-\hat{\Delta})} 2^{-n\left(I(Z ; U)-\gamma_{n}\right)} \rightarrow 0
\end{aligned}
$$

if $\hat{R}-\hat{\Delta}<I(Z ; U)-\gamma_{n}$. The probability of the last event can be bounded as

$$
\begin{aligned}
& \operatorname{Pr}\left(\mathcal{E}_{3} \mid W=1\right) \\
& \leq \operatorname{Pr}\left\{\exists\left(w^{\prime}, l_{w^{\prime}}^{\prime}\right), w^{\prime} \neq 1,\right. \\
& \left.\quad\left(Y^{n}, U^{n}\left(J_{w^{\prime}}\right), V^{n}\left(J_{w^{\prime}}, K_{w^{\prime}}, l_{w^{\prime}}^{\prime}\right)\right) \in \mathcal{T}_{\epsilon}^{n} \mid W=1\right\} \\
& \leq \sum_{i=2}^{2^{n \hat{R}}} \sum_{l_{i}^{\prime}} \operatorname{Pr}\left\{\left(Y^{n}, U^{n}\left(J_{i}\right), V^{n}\left(J_{i}, K_{i}, l_{i}^{\prime}\right)\right) \in \mathcal{T}_{\epsilon}^{n} \mid W=1\right\} .
\end{aligned}
$$

Since for $i=2, \ldots, M$

$$
\begin{aligned}
\operatorname{Pr}\left\{\left(Y^{n},\right.\right. & \left.\left.U^{n}\left(J_{i}\right), V^{n}\left(J_{i}, K_{i}, l_{i}^{\prime}\right)\right) \in \mathcal{T}_{\epsilon}^{n} \mid W=1\right\} \\
& =\sum_{u^{n}, v^{n}} \sum_{y^{n} \in \mathcal{T}_{\epsilon}\left(Y \mid u^{n}, v^{n}\right)} p\left(u^{n}, v^{n}\right) p\left(y^{n} \mid W=1\right) \\
& \leq 2^{-n\left(H(Y)-H(Y \mid U, V)-\gamma_{n}\right)}=2^{-n\left(I(Y ; U, V)-\gamma_{n}\right)},
\end{aligned}
$$

$\operatorname{Pr}\left(\mathcal{E}_{3} \mid W=1\right) \rightarrow 0$ if $\hat{R}+\hat{R}_{V}^{\prime}<I(Y ; U, V)-\gamma_{n}$, where (a) is valid due to the independence of $Y^{n}$ and $W$. Since $\operatorname{Pr}\left(\mathcal{E}_{1} \rightarrow 0\right)$ and $\operatorname{Pr}\left(\mathcal{E}_{3}\right) \rightarrow 0$,

$$
\operatorname{Pr}(\hat{W} \neq W) \rightarrow 0 .
$$

Moreover, due to the union bound

$$
\operatorname{Pr}\{\mathcal{E}\} \rightarrow 0, \text { as } n \rightarrow \infty \text {. }
$$

Due to the typical average lemma [8, p.26], we obtain

$$
\begin{aligned}
\mathbb{E}\left[d\left(X^{n}, \hat{X}^{n}\right)\right] & <\mathbb{E}\left[d\left(X^{n}, \hat{X}^{n}\right) \mid \mathcal{E}^{c}\right]+\operatorname{Pr}(\mathcal{E}) d_{\max } \\
& <D+\gamma_{n}
\end{aligned}
$$

for sufficiently large $n$. In summary, if the following conditions

$$
\begin{gathered}
R_{1}>I(X ; U), \quad R_{2}+R_{V}^{\prime}>I(X ; V \mid U), \\
R_{V}^{\prime}<I(Y ; V \mid U), \\
R<R_{L}+I(Z ; U), \\
R+R_{V}^{\prime}<I(Y ; U, V),
\end{gathered}
$$

hold, then there exists a data processing scheme that satisfies all the requirements for all sufficiently large $n$. By FourierMotzkin elimination [8, Appendix C] and the rate transferring argument, from $R_{2}$ to $R_{1}$, we obtain

$$
\begin{aligned}
R_{1} & \geq I(X ; U), \\
R_{1}+R_{2} & \geq I(X ; U)+I(X ; V \mid U, Y), \\
R_{1}+R_{2}-R & \geq I(X ; U, V \mid Y), \\
R & \leq \min \left\{R_{L}+I(Z ; U), I(Y ; U, V)\right\},
\end{aligned}
$$

since by definition, the achievable region is closed. 


\section{B. Converse}

Given $\epsilon>0$ assume that there exist mappings such that all the conditions are fulfilled for all sufficiently large $n$. Furtheremore, assume that $\frac{1}{n}<\epsilon$. Since $\operatorname{Pr}(\hat{W} \neq W)<\epsilon$, Fano's inequality implies

$$
\begin{gathered}
H\left(W \mid Y^{n}, J_{\mathcal{L}}, K_{\mathcal{L}}\right)<1+\operatorname{Pr}(\hat{W} \neq W) \log M \\
<1+\epsilon \log M .
\end{gathered}
$$

We also establish a variant of Fano's inequality. Define

$$
E= \begin{cases}1 & \text { if } W \in g_{1}\left(Z^{n},\left\{J_{i}\right\}_{i=1}^{M}\right) \\ 0 & \text { otherwise }\end{cases}
$$

Since $W$ is in the list when $E=1$, the error probability is bounded by $P_{e}=\operatorname{Pr}(E=0)<\epsilon$. We expand the conditional entropy $H\left(W, E \mid Z^{n},\left\{J_{i}\right\}_{i=1}^{M}\right)$ in two ways

$$
\begin{gathered}
\begin{array}{c}
H\left(E, W \mid Z^{n},\left\{J_{i}\right\}_{i=1}^{M}\right)=H\left(W \mid Z^{n},\left\{J_{i}\right\}_{i=1}^{M}\right) \\
+\underbrace{H\left(E \mid W, Z^{n},\left\{J_{i}\right\}_{i=1}^{M}\right)}_{0}
\end{array} \\
=H\left(E \mid Z^{n},\left\{J_{i}\right\}_{i=1}^{M}\right)+H\left(W \mid E, Z^{n},\left\{J_{i}\right\}_{i=1}^{M}\right) \\
\leq h\left(P_{e}\right)+\operatorname{Pr}(E=0) H\left(W \mid Z^{n},\left\{J_{i}\right\}_{i=1}^{M}, E=0\right) \\
\quad+\operatorname{Pr}(E=1) H\left(W \mid Z^{n},\left\{J_{i}\right\}_{i=1}^{M}, E=1\right) \\
\leq h\left(P_{e}\right)+P_{e} \log M+n\left(R_{L}+\epsilon\right) \\
=n\left(R_{L}+\epsilon+\frac{1}{n}\left(h\left(P_{e}\right)+P_{e} \log M\right)\right) \leq n\left(R_{L}+\epsilon_{n}\right),
\end{gathered}
$$

where $\epsilon_{n}=2 \epsilon+\frac{1}{n} \epsilon \log M$ and $h(\cdot)$ is the binary entropy function. Define random variables

$$
\begin{aligned}
U_{i} & =\left(W, J_{W}, Y^{i-1}\right), \\
V_{i} & =\left(U_{i}, K_{W}, Y_{i+1}^{n}\right), \quad i \in[1: n] .
\end{aligned}
$$

Observe that $U_{i}-V_{i}-X_{i}(W)-Y_{i}-Z_{i}$ for all $i \in[1: n]$, due to the memoryless of the observational and pre-processing channels. The identification rate can be bounded firstly as

$$
\begin{aligned}
n(R-\epsilon) & \leq \log M=H(W) \\
& =I\left(W ; Z^{n},\left\{J_{i}\right\}_{i=1}^{M}\right)+H\left(W \mid Z^{n},\left\{J_{i}\right\}_{i=1}^{M}\right) \\
& \stackrel{(\star)}{\leq} I\left(W ; Z^{n} \mid\left\{J_{i}\right\}_{i=1}^{M}\right)+n\left(R_{L}+\epsilon_{n}\right) \\
& \leq I\left(W,\left\{J_{i}\right\}_{i=1}^{M} ; Z^{n}\right)+n\left(R_{L}+\epsilon_{n}\right) \\
& =I\left(W, J_{W} ; Z^{n}\right)+n\left(R_{L}+\epsilon_{n}\right) \\
& =\sum_{i=1}^{n} I\left(W, J_{W}, Z^{i-1} ; Z_{i}\right)+n\left(R_{L}+\epsilon_{n}\right), \\
& \stackrel{(a)}{\leq} \sum_{i=1}^{n} I\left(W, J_{W}, Y^{i-1} ; Z_{i}\right)+n\left(R_{L}+\epsilon_{n}\right),
\end{aligned}
$$

where $(\star)$ holds since $W$ is independent of $\left\{J_{i}\right\}_{i=1}^{M}$. (a) holds due to the Markov chain $Z^{i-1}-Y^{i-1}-\left(Z_{i}, W, J_{W}\right)$ for all $i \in[1: n]$, due to the memoryless of the pre-processing. This implies that

$$
(R-\epsilon)(1-\epsilon) \leq \frac{1}{n} \sum_{i=1}^{n} I\left(U_{i} ; Z_{i}\right)+R_{L}+2 \epsilon .
$$

Secondly,

$$
\begin{aligned}
n(R & -\epsilon) \leq \log M=H(W) \\
& =I\left(W ; Y^{n}, J_{\mathcal{L}}, K_{\mathcal{L}}\right)+H\left(W \mid Y^{n}, J_{\mathcal{L}}, K_{\mathcal{L}}\right) \\
& \stackrel{(b)}{\leq} I\left(W ; Y^{n}, Z^{n},\left\{J_{i}\right\}_{i=1}^{M},\left\{K_{i}\right\}_{i=1}^{M}\right)+1+\epsilon \log M \\
& \stackrel{(c)}{=} I\left(W ; Y^{n},\left\{J_{i}\right\}_{i=1}^{M},\left\{K_{i}\right\}_{i=1}^{M}\right)+1+\epsilon \log M \\
& \stackrel{(\star \star)}{\leq} I\left(W,\left\{J_{i}\right\}_{i=1}^{M},\left\{K_{i}\right\}_{i=1}^{M} ; Y^{n}\right)+1+\epsilon \log M \\
& \leq \sum_{i=1}^{n} I\left(W, J_{W}, K_{W}, Y^{n \backslash i} ; Y_{i}\right)+1+\epsilon \log M,
\end{aligned}
$$

where (b) holds since $\mathcal{L}=g_{1}\left(Z^{n},\left\{J_{i}\right\}_{i=1}^{M}\right)$, hence $J_{\mathcal{L}}$ can be thought as a function of $\left\{J_{i}\right\}_{i=1}^{M}$ and $Z^{n}$. Similarly, $K_{\mathcal{L}}$ can be also viewed as a function of $\left\{J_{i}\right\}_{i=1}^{M},\left\{K_{i}\right\}_{i=1}^{M}$ and $Z^{n}$. We also use the inequality (25) in $(b)$. (c) is valid due to the Markov chain $Z^{n}-Y^{n}-\left(W,\left\{J_{i}\right\}_{i=1}^{M},\left\{K_{i}\right\}_{i=1}^{M}\right)$. ( $\left.\star \star\right)$ holds since $W$ is independent of $\left\{J_{i}\right\}_{i=1}^{M}$ and $\left\{K_{i}\right\}_{i=1}^{M}$. Using (28) and (31) gives us

$$
(R-\epsilon)(1-\epsilon) \leq \frac{1}{n} \sum_{i=1}^{n} I\left(U_{i}, V_{i} ; Y_{i}\right)+\epsilon .
$$

Furthermore, the sum compressed rate can be bounded as

$$
\begin{aligned}
n\left(R_{1}+\right. & \left.R_{2}+\epsilon\right) \geq H\left(J_{W}, K_{W} \mid W\right) \\
\geq & I\left(X^{n}(W), Y^{n} ; J_{W}, K_{W} \mid W\right) \\
\geq & I\left(Y^{n} ; J_{W} \mid W\right)+I\left(X^{n}(W) ; J_{W}, K_{W} \mid W, Y^{n}\right) \\
= & \sum_{i=1}^{n}\left(I\left(Y_{i} ; W, J_{W}, Y^{i-1}\right)\right. \\
& \left.\quad+I\left(X_{i}(W) ; W, J_{W}, K_{W}, Y^{n \backslash i}, X^{i-1}(W) \mid Y_{i}\right)\right) \\
\geq & \sum_{i=1}^{n}\left(I\left(X_{i}(W), Y_{i} ; W, J_{W}, Y^{i-1}\right)\right. \\
& \left.\quad+I\left(X_{i}(W) ; K_{W}, Y_{i+1}^{n} \mid Y_{i}, W, J_{W}, Y^{i-1}\right)\right) \\
& \stackrel{(d)}{=} \sum_{i=1}^{n} I\left(X_{i}(W) ; U_{i}\right)+I\left(X_{i}(W) ; V_{i} \mid U_{i}, Y_{i}\right),
\end{aligned}
$$

where $(d)$ follows from the Markov chain $Y_{i}-X_{i}(W)-$ $\left(W, J_{W}, Y^{i-1}\right)$ for all $i \in[1: n]$. Similarly we can show that

$$
n\left(R_{1}+\epsilon\right) \geq H\left(J_{W} \mid W\right) \geq \sum_{i=1}^{n} I\left(X_{i}(W) ; U_{i}\right) .
$$

In addition,

$$
\begin{aligned}
& n\left(R_{1}+R_{2}+\epsilon\right)-\log M \\
& \geq H\left(J_{W}, K_{W} \mid W\right)-I\left(W, J_{W}, K_{W} ; Y^{n}\right)-(1+\epsilon \log M) \\
& \geq I\left(X^{n}(W) ; J_{W}, K_{W}, W\right) \\
& \quad \quad-I\left(Y^{n} ; J_{W}, K_{W}, W\right)-(1+\epsilon \log M) \\
& \stackrel{(*)}{=} I\left(X^{n}(W) ; J_{W}, K_{W}, W \mid Y^{n}\right)-(1+\epsilon \log M)
\end{aligned}
$$




$$
\geq \sum_{i=1}^{n} I\left(X_{i}(W) ; U_{i}, V_{i} \mid Y_{i}\right)-(1+\epsilon \log M),
$$

where $(*)$ holds due to the memoryless property of the observational channel, i.e., $Y^{n}-X^{n}(W)-\left(W, J_{W}, K_{W}\right)$. This leads to

$$
\begin{aligned}
R_{1}+R_{2} & +2 \epsilon-(R-\epsilon)(1-\epsilon) \\
& \geq \frac{1}{n} \sum_{i=1}^{n} I\left(X_{i}(W) ; U_{i}, V_{i} \mid Y_{i}\right) .
\end{aligned}
$$

Since

$$
\begin{aligned}
D+\epsilon & >\mathbb{E}\left[d\left(X^{n}(W), g_{3}\left(J_{\hat{W}}, K_{\hat{W}}, Y^{n}\right)\right)\right] \\
& >\operatorname{Pr}(\hat{W}=W) \times \\
& \mathbb{E}\left[d\left(X^{n}(W), g_{3}\left(J_{W}, K_{W}, Y^{n}\right) \mid \hat{W}=W\right],\right.
\end{aligned}
$$

the following chain of expressions holds

$$
\begin{aligned}
& \mathbb{E}\left[d\left(X^{n}(W), g_{3}^{\prime}\left(W, J_{W}, K_{W}, Y^{n}\right)\right)\right] \\
& =\mathbb{E}\left[d\left(X^{n}(W), g_{3}\left(J_{W}, K_{W}, Y^{n}\right)\right)\right] \\
& \leq \mathbb{E}\left[d\left(X^{n}(W), g_{3}\left(J_{W}, K_{W}, Y^{n}\right)\right) \mid \hat{W}=W\right] \operatorname{Pr}(\hat{W}=W) \\
& +\operatorname{Pr}(\hat{W} \neq W) d_{\max }<D+\left(1+d_{\max }\right) \epsilon .
\end{aligned}
$$

Let $Q$ be a uniform random variable on $[1: n]$ and independent of everything else. Define $U=\left(U_{Q}, Q\right), V=\left(V_{Q}, Q\right)$, and

$$
f\left(U, V, Y_{Q}\right)=g_{3 Q}^{\prime}\left(W, J_{W}, K_{W}, Y^{n}\right) .
$$

Note that $U-V-X_{Q}(W)-Y_{Q}-Z_{Q}$ still holds. Then the distortion constraint can be rewritten as

$$
D+\left(1+d_{\max }\right) \epsilon>\mathbb{E}\left[d\left(X_{Q}(W), f\left(U, V, Y_{Q}\right)\right)\right]
$$

Since $\left(X_{Q}(W), Y_{Q}, Z_{Q}\right)$ has the same joint distribution as $(X, Y, Z),\left((R-\epsilon)(1-\epsilon), R_{1}+\epsilon, R_{2}+\epsilon, R_{L}+2 \epsilon, D+(1+\right.$ $\left.\left.d_{\text {max }}\right) \epsilon\right) \in \mathcal{R}^{\star}$ by the cardinality bounding arguments. Taking $\epsilon \rightarrow 0$ completes the backward direction.

\section{TWO RELATED PROBLEMS}

When the distortion level $D=d_{\max }$, i.e., the distortion constraint can be removed, then binning for the second layer, described by $V$, is not necessary. We obtain the following corollary.

Corollary 1. The rate region for our identification, i.e., $D=$ $d_{\text {max }}$, is given by the set of tuples $\left(R, R_{1}, R_{2}, R_{L}\right)$ such that

$$
\begin{aligned}
U & -V-X-Y-Z, \\
R_{1} & \geq I(X ; U), \quad R_{1}+R_{2} \geq I(X ; U, V), \\
R & \leq \min \left\{R_{L}+I(Z ; U), I(Y ; U, V)\right\},
\end{aligned}
$$

where $U$ and $V$ are random variables taking values on alphabets $\mathcal{U}$ and $\mathcal{V}$, respectively, with $|\mathcal{U}| \leq|\mathcal{X}|+4$ and $|\mathcal{V}| \leq(|\mathcal{X}|+4)(|\mathcal{X}|+1)$

A related problem is stated the following. The data sequence $x^{n}(w)$ is observed through the channel $p_{Z Y \mid X}$ by two observers 1 and 2, which obtain $y^{n}$, and $z^{n}$, respectively. Moreover, observer 2 has access only to the information stored in the first layer and is interested in obtaining a list of users in the database only, for instance due to time constraint or due to privilege restriction, i.e., the decoding mapping and the requirement for the second user are given by

$$
\mathcal{L}=g_{2}\left(z^{n},\left\{j_{i}\right\}_{i=1}^{M}\right), \text { and } \operatorname{Pr}(W \notin \mathcal{L})<\epsilon,
$$

where $|\mathcal{L}| \leq 2^{n \Delta}$. In contrast to observer 2 , observer 1 has access to both layers and wants to identify the user correctly, i.e., the decoding mapping and the requirement of the first observer are

$$
\hat{w}=g_{1}\left(y^{n},\left\{j_{i}\right\}_{i=1}^{M},\left\{k_{i}\right\}_{i=1}^{M}\right), \text { and } \operatorname{Pr}(W \neq \hat{W})<\epsilon .
$$

In other words, the identification processes for two observers are separated. The rate region for this problem can be described by the following proposition.

Proposition 1. The rate region for the stated problem is the set of tuples $\left(R, R_{1}, R_{2}, R_{L}\right)$ such that

$$
\begin{aligned}
U & -V-X-(Y, Z), \\
R_{1} & \geq I(X ; U), \quad R_{1}+R_{2} \geq I(X ; U, V), \\
R & \leq \min \left\{R_{L}+I(Z ; U), I(Y ; U, V)\right\},
\end{aligned}
$$

where $U$ and $V$ are random variables taking values on finite alphabets $\mathcal{U}$ and $\mathcal{V}$, respectively, with $|\mathcal{U}| \leq|\mathcal{X}|+4$ and $|\mathcal{V}| \leq$ $(|\mathcal{X}|+4)(|\mathcal{X}|+1)$.

Note that the Markov condition $X-Y-Z$ is not needed since two processes work independently. This means that our problem can be viewed as a partial cooperation scheme between two identification processes.

\section{ACKNOWLEDGMENT}

This work is partially supported by Swedish Research Council (VR), project grant 2016-03853, and the PITA project of SRA ICT-TNG funded by the Swedish government.

\section{REFERENCES}

[1] F. Willems, T. Kalker, and J.-P. Linnartz, "On the capacity of a biometrical identification system," in 2003 IEEE International Symposium on Information Theory. IEEE, p. 82.

[2] E. Tuncel, "Capacity/storage tradeoff in high-dimensional identification systems," IEEE Trans. Inf. Theory, vol. 55, no. 5, pp. 2097-2106, 2009.

[3] E. Tuncel and D. Gündüz, "Identification and lossy reconstruction in noisy databases," IEEE Trans. Inf. Theory, vol. 60, no. 2, pp. 822-831, 2014.

[4] F. M. Willems, "Searching methods for biometric identification systems: Fundamental limits," in 2009 IEEE International Symposium on Information Theory. IEEE, 2009, pp. 2241-2245.

[5] E. Tuncel, "Recognition capacity versus search speed in noisy databases," in Information Theory Proceedings (ISIT), 2012 IEEE International Symposium on. IEEE, 2012, pp. 2566-2570.

[6] F. Farhadzadeh and F. M. Willems, "Identification rate, search and memory complexity tradeoff: Fundamental limits," IEEE Trans. Inf. Theory, vol. 62, no. 11, pp. 6173-6188, 2016.

[7] E. Tuncel, P. Koulgi, and K. Rose, "Rate-distortion approach to databases: Storage and content-based retrieval," IEEE Trans. Inf. Theory, vol. 50, no. 6, pp. 953-967, 2004.

[8] A. El Gamal and Y.-H. Kim, Network information theory. Cambridge university press, 2011.

[9] M. T. Vu, T. Oechtering, and M. Skoglund, "Hierarchical identification with pre-processing," Manuscript in preparation. 